\title{
Evaluation of Different Fungicides against Macrophomina phaseolina (Tassi) Goid. Causing Dry Root Rot of Chickpea (Cicer arietinum L.) invitro
}

\author{
R. Lokesh*, K. B. Rakholiya and M. R. Thesiya \\ Department of Plant Pathology, N. M. College of Agriculture, \\ Navsari Agricultural University, Navsari, Gujarat 396 450, India \\ *Corresponding author
}

\begin{tabular}{|l|}
\hline Ke y w or d s \\
Chickpea, dry root \\
rot, in vitro, \\
$\begin{array}{l}\text { Macrophomina } \\
\text { phaseolina, per cent } \\
\text { growth inhibition, } \\
\text { fungicides }\end{array}$ \\
\hline Article Info \\
\hline $\begin{array}{l}\text { Accepted: } \\
\text { 11 June } 2020 \\
\text { Available Online: } \\
\text { 10 July } 2020\end{array}$ \\
\hline
\end{tabular}

\begin{abstract}
Chickpea (Cicer arietinum L.) is one of the major legume pulse crops and it is majorly grown in India and other semi-arid regions of the world. Dry root rot of chickpea is the most destructive disease of chickpea. Therefore, in present studies fungicides (six systemic, seven non systemic and six ready mix fungicides) were tested in vitro at three different concentrations by poisoned food technique for evaluating their efficacy against $M$. phaseolina. Among systemic fungicides, significantly highest average mycelial growth inhibition was with carbendazim $(85.88 \%)$, thiophanate methyl $(84.32 \%)$ followed by hexaconazole (75.29\%) was also considerably effective fungicide but tebuconazole (47.06\%), azoxystrobin (36.08\%), propiconazole $(36.86 \%)$ proved comparatively less in their efficacy against M. phaseolina. Among nonsystemic fungicides, significantly highest average mycelial growth inhibition over control was recorded in mancozeb (90.20\%), followed by chlorothalonil (88.24\%). The remaining fungicides viz., zineb (78.04\%), thiram $(76.47 \%)$, and propineb $(49.41 \%)$, were moderately effective. While sulphur $(23.53 \%)$ recorded least effective in growth inhibition as compared to other fungicides against $M$. phaseolina. Among ready mix fungicides, significantly highest per cent growth inhibition over control was recorded $(88.24 \%)$ in carbendazim $12 \%+$ mancozeb $63 \%$ followed by carboxin $37.5 \%+$ thiram $37.5 \%$, $(83.14 \%)$. The remaining fungicides viz., pyraclostrobin $5 \%+$ mitiram $55 \%(70.59 \%)$, captan $70 \%+$ hexaconazole $5 \%$ $(76.47 \%)$ and pyraclostrobin $5 \%+$ mitiram $55 \%(59.21 \%)$ were moderately effective. While pyraclostrobin $12.5 \%$ + epoxiconazole $4.7 \%$, (38.82\%) and hexaconazole $4 \%$ + zineb $68 \%(27.45 \%)$, recorded least effective in growth inhibition as compared to other fungicides against M. phaseolina.
\end{abstract}

\section{Introduction}

Chickpea (Cicer arietinum L.) is one of the major legume pulse crops and it is majorly grown in India and other semi-arid regions of the world. It belongs to the family Fabaceae, subfamily Faboideae. Chickpea is a selfpollinating diploid crop with chromosome number $(2 n=16)$ and a genome consisting of $740 \mathrm{Mbp}$. It is documented to have originated in south-eastern Turkey from where it has spread to other countries of the world. Among the major pulse crops, chickpea contributes nearly 32.6 per cent and 40.5 per cent of total pulse area and total pulse production, respectively.

In India, chickpea is cultivated in an area of about 8.32 million ha with a production of 9.8metrictone and $925 \mathrm{~kg} / \mathrm{ha}$ productivity (Anonymous, 2018). The chickpea crop was reported to be attacked by nearly 172 
pathogens (67 fungi, 22 viruses, 3 bacteria, 80 nematodes) from all over the world (Nene $e t$ al., 1996). Some of the serious diseases in chickpea are of dry and wet root rot [Rhizoctonia bataticola, (Taub.) Butler], wilt [Fusarium oxysporum f. sp. ciceri, (Padwick) Snyd. \& Hans.] ascochyta blight [Ascocthya rabiei, (Pass.) Labr.] and collar rot (Sclerotium rolfsii Sacc.). Among the diseases of chickpea, dry root rot has an emerging the most destructive and constraint to chickpea productivity and production, as the disease is more prevalent during hot temperature of 30 to $35^{\circ} \mathrm{C}$ and low soil moisture conditions (Taya et al., 1988; Pande and Sharma, 2010). $R$. bataticola is a soil-inhabiting pathogen and capable of infecting chickpea at any crop stage, but most commonly infects chickpea at post-reproductive stage in dry and warm regions (Sharma and Pande, 2013).

\section{Materials and Methods}

\section{Isolation of pathogens}

Diseased specimens were to be brought to laboratory and examined under microscope for preliminary examination. Isolation of pathogen, small pieces of infected samples were cut from the diseased portion along with some healthy tissues and surface sterilized with 0.1 per cent mercuric chloride solution for 1minute followed by three washing with sterilized distilled water. The surface sterilized pieces were transferred to $20 \mathrm{ml}$ poured potato dextrose agar (PDA) plates and incubated at $27 \pm 2{ }^{\circ} \mathrm{C}$. After seven days of incubation, the fungal growth was transferred aseptically on PDA slants and purified following hyphal tip method.

\section{In vitro evaluation of fungicides}

Efficacy of seven non-systemic fungicides and six systemic and ready-mix fungicides was evaluated in vitro at various concentrations against $M$. phaseolina, applying Poisoned food technique (Dhingra and Sinclair, 1995) and using PDA as basal culture medium. Systemic fungicides at the rate 50, 250 and $500 \mathrm{ppm}$ while non-systemic at the rate 1000, 2000 and 2500 and readymix fungicides at the rate 500, 1500 and 2000 ppm. Based on active ingredient, requisite quantity of the fungicide was mixed in $100 \mathrm{ml}$ potato dextrose agar medium in $250 \mathrm{ml}$ flask and well shaken to facilitate uniform mixture of fungicides and $20 \mathrm{ml}$ was poured in each sterilized plate (90 $\mathrm{mm}$ diameter). After $24 \mathrm{~h}$ a disc of five $\mathrm{mm}$ was placed in the centre of each poured plate. The discs were cut with the help of a sterilized cork borer from 10 days old culture of $M$. phaseolina Inoculated plates were incubated at $27 \pm 1{ }^{\circ} \mathrm{C}$. The colony growth was measured after 24 hrs interval till the entire plate of control treatment was completely covered with mycelium. Suitable check was maintained without fungicide and inoculated with $M$. phaseolina. The per cent growth inhibition (PGl) over control was calculated using the following formula.

$$
\text { PGI }(\%)=\frac{C-T}{C} \times 100
$$

Where, PGI $(\%)=$ Per cent growth inhibition $\mathrm{C}=$ Average diameter $(\mathrm{mm})$ of mycelial colony of control plate

$\mathrm{T}=$ Average diameter $(\mathrm{mm})$ of mycelial colony of treated plate in treated plates

\section{Results and Discussion}

\section{Effect of different systemic fungicides on growth inhibition of $M$. phaseolina}

Effect of these fungicides on radial growth and inhibition of test pathogen were recorded. All the treatments were replicated thrice and a suitable untreated control (without fungicide) was also maintained. 
Results (Plate 1 and Table 1) revealed that the fungicides tested significantly inhibited growth of the test fungus over untreated control. Further, it was found that per cent inhibition of the test pathogen was increased with the increase in concentration of the fungicides tested. At $50 \mathrm{ppm}$ concentration, significantly highest per cent growth inhibition over control was recorded in carbendazim $(82.35 \%)$ followed by thiophanate methyl (78.82\%). At $250 \mathrm{ppm}$ concentration, propiconazole recorded significantly highest per cent growth inhibition $(74.44 \%)$ followed by carbendazim (85.88\%). At 500 ppm concentration, cent per cent growth inhibition of the pathogen recorded by carbendazim $(90.59 \%)$ and followed by thiophanate methyl $(84.71 \%)$.Thus, both the fungicides proved the most effective for $M$. Phaseolina. Next best fungicide in order of merit was hexaconazole. While, the rest of the fungicides were comparatively medium or less effective against $M$. Phaseolina. Azoxystrobin was found least effective at all concentration as compared to other fungicides. The similar results were found and recorded by Chattopadhyay and Kalpana (2002), Khan and Gangopadhyay (2008) Kumari and Shekhawat (2012) and Ebenezar and Wesely (2000) found that carbendazim at $0.1 \%$ completely inhibited the growth of $M$. phaseolina.

\section{Effects of non-systemic fungicides on the growth of $M$. phaseolina}

Results (Plate 2 and Table 2) revealed that all non-systemic tested significantly inhibited mycelial growth of $M$. phaseolina, over untreated control. At 1000 ppm concentration, significantly highest per cent growth inhibition over control was recorded in mancozeb (82.74\%) followed by chlorothalonil (78.82\%), zineb (70.59\%), thiram $(64.71 \%)$ and propineb $(41.56 \%)$ were moderately effective. While sulphur (12.15\%) recorded least effective in growth inhibition. At 2000 ppm concentration, mancozeb $(85.88 \%)$ followed by chlorothalonil $(83.92 \%)$, the remaining fungicides viz., zineb (72.55\%), thiram (70.20\%). At $2500 \mathrm{ppm}$ concentration mancozeb (90.20\%), followed by chlorothalonil $(88.24 \%)$. The remaining fungicides viz., zineb $(78.04 \%)$, thiram $(76.47 \%)$, and propineb $(49.41 \%)$ were moderately effective. While sulphur $(23.53 \%)$ recorded least effective in growth inhibition as compared to other fungicides against $M$. phaseolina.

Thus, both the fungicides proved the most effective for $M$. phaseolina. The present findings are confirmed with the results Ravichandran and Hedge (2017) observed that the fungicides chlorothalonil and mancozeb at 0.2 per cent were effective. Maruti et al., (2017) reported that mancozeb and thiram showed 100 per cent inhibition at 0.3 per cent concentration. Sangappa and Mallesh (2016) recorded that the fungicides thiophanate methyl and triadimefon showed 100 per cent mycelia inhibition at $0.1,0.2$ and $0.3 \%$.

\section{Effect of ready-mix fungicides on growth inhibition of $M$. phaseolina}

Results (Plate 3 and Table 3 ) revealed that all ready-mix fungicides tested significantly inhibited mycelial growth of $M$. phaseolina, over untreated control. Further, per cent mycelial inhibition was increased with increase in concentrations of the fungicides tested. At 500 ppm concentration, highest per cent growth inhibition over control was recorded $(82.35 \%)$ in carbendazim $12 \%+$ mancozeb $63 \%$ followed by carboxin $37.5 \%+$ thiram $37.5 \% \quad(78.82 \%)$. The remaining fungicides viz., pyraclostrobin $5 \%+$ mitiram $55 \%(65.09 \%)$, captan $70 \%+$ hexaconazole $5 \%(62.74 \%)$ against $M$. phaseolina. 
Table.1 Evaluation of systemic fungicides against M. phaseolina in vitro

\begin{tabular}{|c|c|c|c|c|}
\hline Tr. No & $\begin{array}{l}\text { Technical name } \\
\text { of fungicides }\end{array}$ & $\begin{array}{l}\text { Concentration } \\
(\text { ppm) }\end{array}$ & $\begin{array}{l}\text { Average colony diameter } \\
(\mathbf{m m}) @\end{array}$ & $\begin{array}{l}\text { Per cent growth } \\
\text { inhibition }\end{array}$ \\
\hline \multirow[t]{3}{*}{$\mathbf{T}_{1}$} & \multirow[t]{3}{*}{$\begin{array}{l}\text { Carbendazim } \\
(50 \mathrm{WP})\end{array}$} & 50 & $\begin{array}{c}3.92 \\
(15.00)\end{array}$ & 82.35 \\
\hline & & 250 & $\begin{array}{c}3.52 \\
(12.00)\end{array}$ & 85.88 \\
\hline & & 500 & $\begin{array}{c}2.88 \\
(8.00)\end{array}$ & 90.59 \\
\hline \multirow[t]{3}{*}{$\mathbf{T}_{2}$} & \multirow[t]{3}{*}{$\begin{array}{l}\text { Tebuconazole } \\
\text { (25.9EC) }\end{array}$} & 50 & $\begin{array}{c}6.96 \\
(48.00)\end{array}$ & 43.53 \\
\hline & & 250 & $\begin{array}{c}6.74 \\
(45.00)\end{array}$ & 47.06 \\
\hline & & 500 & $\begin{array}{c}6.59 \\
(43.00)\end{array}$ & 49.41 \\
\hline \multirow[t]{3}{*}{$\mathbf{T}_{3}$} & \multirow[t]{3}{*}{$\begin{array}{l}\text { Propiconazole } \\
\text { (25 EC) }\end{array}$} & 50 & $\begin{array}{c}7.67 \\
(58.33)\end{array}$ & 31.38 \\
\hline & & 250 & $\begin{array}{c}7.36 \\
(53.67)\end{array}$ & 36.86 \\
\hline & & 500 & $\begin{array}{c}7.06 \\
(49.33)\end{array}$ & 41.96 \\
\hline \multirow[t]{3}{*}{$\mathbf{T}_{4}$} & \multirow[t]{3}{*}{$\begin{array}{l}\text { Azoxystrobin } \\
(23 \text { SC) }\end{array}$} & 50 & $\begin{array}{c}8.27 \\
(68.00)\end{array}$ & 20.00 \\
\hline & & 250 & $\begin{array}{c}7.40 \\
(54.33)\end{array}$ & 36.08 \\
\hline & & 500 & $\begin{array}{c}7.24 \\
(52.00)\end{array}$ & 38.82 \\
\hline \multirow[t]{3}{*}{$\mathbf{T}_{5}$} & \multirow[t]{3}{*}{$\begin{array}{l}\text { Hexaconazole } \\
\text { (5 EC) }\end{array}$} & 50 & $\begin{array}{c}4.98 \\
(24.33)\end{array}$ & 71.38 \\
\hline & & 250 & $\begin{array}{c}4.63 \\
(21.00)\end{array}$ & 75.29 \\
\hline & & 500 & $\begin{array}{c}4.17 \\
(17.00)\end{array}$ & 80.00 \\
\hline \multirow[t]{3}{*}{$\mathbf{T}_{6}$} & \multirow[t]{3}{*}{$\begin{array}{l}\text { Thiophanate } \\
\text { methyl ( } 70 \mathrm{WP})\end{array}$} & 50 & $\begin{array}{c}4.29 \\
(18.00)\end{array}$ & 78.82 \\
\hline & & 250 & $\begin{array}{c}3.71 \\
(13.33)\end{array}$ & 84.32 \\
\hline & & 500 & $\begin{array}{c}3.67 \\
(13.00)\end{array}$ & 84.71 \\
\hline $\mathbf{T}_{7}$ & Control & & $\begin{array}{c}9.25 \\
(85.00)\end{array}$ & \\
\hline \multicolumn{3}{|c|}{ S. Em. \pm} & 0.17 & \\
\hline \multicolumn{3}{|c|}{ C. D. at $5 \%$} & 0.50 & \\
\hline \multicolumn{3}{|c|}{ C. V. (\%) } & 4.71 & \\
\hline
\end{tabular}


Int.J.Curr.Microbiol.App.Sci (2020) 9(7): 901-911

Table.2 Evaluation of non-systemic fungicides against $M$. phaseolina in vitro

\begin{tabular}{|c|c|c|c|c|}
\hline Tr. no. & $\begin{array}{l}\text { Technical name of } \\
\text { fungicides }\end{array}$ & Concentration (ppm) & $\begin{array}{l}\text { Average colony } \\
\text { diameter } \\
(\mathbf{m m}) @\end{array}$ & $\begin{array}{l}\text { Per cent growth } \\
\text { inhibition }\end{array}$ \\
\hline \multirow[t]{3}{*}{$\mathbf{T}_{1}$} & \multirow[t]{3}{*}{$\begin{array}{l}\text { Copperoxychloride } \\
\text { (50WP) }\end{array}$} & 1000 & $\begin{array}{c}7.40 \\
(54.33)\end{array}$ & 36.08 \\
\hline & & 2000 & $\begin{array}{c}6.51 \\
(42.00)\end{array}$ & 50.59 \\
\hline & & 2500 & $\begin{array}{c}6.11 \\
(37.00)\end{array}$ & 56.47 \\
\hline \multirow[t]{3}{*}{$\mathbf{T}_{2}$} & \multirow[t]{3}{*}{ Mancozeb (75WP) } & 1000 & $\begin{array}{c}3.89 \\
(14.67)\end{array}$ & 82.74 \\
\hline & & 2000 & $\begin{array}{c}3.53 \\
(12.00)\end{array}$ & 85.88 \\
\hline & & 2500 & $\begin{array}{c}2.96 \\
(8.33)\end{array}$ & 90.20 \\
\hline \multirow[t]{3}{*}{$\mathbf{T}_{3}$} & \multirow[t]{3}{*}{$\begin{array}{l}\text { Chlorothalonil } \\
\text { (75WP) }\end{array}$} & 1000 & $\begin{array}{c}4.30 \\
(18.00)\end{array}$ & 78.82 \\
\hline & & 2000 & $\begin{array}{c}3.76 \\
(13.67)\end{array}$ & 83.92 \\
\hline & & 2500 & $\begin{array}{c}3.23 \\
(10.00)\end{array}$ & 88.24 \\
\hline \multirow[t]{3}{*}{$\mathbf{T}_{4}$} & \multirow[t]{3}{*}{$\begin{array}{l}\text { Propineb } \\
\text { (70WP) }\end{array}$} & 1000 & $\begin{array}{c}7.08 \\
(49.67)\end{array}$ & 41.56 \\
\hline & & 2000 & $\begin{array}{c}7.01 \\
(48.67)\end{array}$ & 42.74 \\
\hline & & 2500 & $\begin{array}{c}6.59 \\
(43.00)\end{array}$ & 49.41 \\
\hline \multirow[t]{3}{*}{$\mathbf{T}_{5}$} & \multirow[t]{3}{*}{$\begin{array}{l}\text { Thiram } \\
\text { (75WP) }\end{array}$} & 1000 & $\begin{array}{c}5.52 \\
(30.00)\end{array}$ & 64.71 \\
\hline & & 2000 & $\begin{array}{c}5.08 \\
(25.33)\end{array}$ & 70.20 \\
\hline & & 2500 & $\begin{array}{c}4.52 \\
(20.00)\end{array}$ & 76.47 \\
\hline \multirow[t]{3}{*}{$T_{6}$} & \multirow[t]{3}{*}{$\begin{array}{l}\text { Zineb } \\
\text { (75WP) }\end{array}$} & 1000 & $\begin{array}{c}5.04 \\
(25.00)\end{array}$ & 70.59 \\
\hline & & 2000 & $\begin{array}{c}4.87 \\
(23.33)\end{array}$ & 72.55 \\
\hline & & 2500 & $\begin{array}{c}4.38 \\
(18.67)\end{array}$ & 78.04 \\
\hline \multirow[t]{3}{*}{$\mathbf{T}_{7}$} & \multirow[t]{3}{*}{$\begin{array}{l}\text { Sulphur } \\
\text { (80 WP) }\end{array}$} & 1000 & $\begin{array}{c}8.67 \\
(74.67)\end{array}$ & 12.15 \\
\hline & & 2000 & $\begin{array}{c}8.38 \\
(69.67)\end{array}$ & 18.04 \\
\hline & & 2500 & $\begin{array}{c}8.09 \\
(65.00)\end{array}$ & 23.53 \\
\hline $\mathbf{T}_{8}$ & Control & & $\begin{array}{c}9.25 \\
(85.00)\end{array}$ & \\
\hline \multicolumn{3}{|c|}{ S. Em. \pm} & 0.15 & \\
\hline \multicolumn{3}{|c|}{ C. D. at $5 \%$} & 0.44 & \\
\hline \multicolumn{3}{|c|}{ C. V. \% } & 4.23 & \\
\hline
\end{tabular}


Table.3 Evaluation of ready-mix fungicides against M. phaseolina in vitro

\begin{tabular}{|c|c|c|c|c|}
\hline $\begin{array}{l}\text { Tr. } \\
\text { no. }\end{array}$ & Technical name of fungicides & $\begin{array}{l}\text { Concentratio } \\
\mathbf{n}(\mathbf{p p m})\end{array}$ & $\begin{array}{l}\text { Average colony } \\
\text { diameter }(\mathbf{m m}) @\end{array}$ & $\begin{array}{l}\text { Per cent growth } \\
\text { inhibition }\end{array}$ \\
\hline \multirow[t]{3}{*}{$\mathbf{T}_{1}$} & \multirow[t]{3}{*}{$\begin{array}{l}\text { Carbendazim } \\
\text { Mancozeb }(63 \%)\end{array}$} & 500 & $\begin{array}{c}3.93 \\
(15.00)\end{array}$ & 82.35 \\
\hline & & 1500 & $\begin{array}{c}3.53 \\
(12.00)\end{array}$ & 85.88 \\
\hline & & 2000 & $\begin{array}{c}3.23 \\
(10.00)\end{array}$ & 88.24 \\
\hline \multirow[t]{3}{*}{$\mathbf{T}_{2}$} & \multirow[t]{3}{*}{$\begin{array}{l}\text { Carboxin }(37.5 \%)+\text { Thiram } \\
(37.5 \%)\end{array}$} & 500 & $\begin{array}{c}4.28 \\
(18.00)\end{array}$ & 78.82 \\
\hline & & 1500 & $\begin{array}{c}3.84 \\
(14.33)\end{array}$ & 83.14 \\
\hline & & 2000 & $\begin{array}{c}3.34 \\
(10.67)\end{array}$ & 87.45 \\
\hline \multirow[t]{3}{*}{$\mathbf{T}_{3}$} & \multirow[t]{3}{*}{$\begin{array}{l}\text { Captan }(50 \%)+\text { Hexaconazole } \\
(5 \%)\end{array}$} & 500 & $\begin{array}{c}5.67 \\
(31.67)\end{array}$ & 62.74 \\
\hline & & 1500 & $\begin{array}{c}5.01 \\
(24.67)\end{array}$ & 70.98 \\
\hline & & 2000 & $\begin{array}{c}4.52 \\
(20.00)\end{array}$ & 76.47 \\
\hline \multirow[t]{3}{*}{$\mathbf{T}_{4}$} & \multirow[t]{3}{*}{$\begin{array}{l}\text { Pyraclostrobin }(12.5 \%) \quad+ \\
\text { Epoxiconazole }(4.7 \%),\end{array}$} & 500 & $\begin{array}{c}6.39 \\
(40.33)\end{array}$ & 52.55 \\
\hline & & 1500 & $\begin{array}{c}5.95 \\
(35.00)\end{array}$ & 58.82 \\
\hline & & 2000 & $\begin{array}{c}5.93 \\
(34.67)\end{array}$ & 59.21 \\
\hline \multirow[t]{3}{*}{$\mathbf{T}_{5}$} & \multirow[t]{3}{*}{$\begin{array}{l}\text { Hexaconazole }(4 \%)+\text { Zineb } \\
(68 \%)\end{array}$} & 500 & $\begin{array}{c}8.11 \\
(65.33)\end{array}$ & 23.14 \\
\hline & & 1500 & $\begin{array}{c}7.88 \\
(61.67)\end{array}$ & 27.45 \\
\hline & & 2000 & $\begin{array}{c}7.24 \\
(52.00)\end{array}$ & 38.82 \\
\hline \multirow[t]{3}{*}{$T_{6}$} & \multirow[t]{3}{*}{$\begin{array}{l}\text { Pyraclostrobin }(5 \%)+\text { Mitiram } \\
(55 \%)\end{array}$} & 500 & $\begin{array}{c}5.49 \\
(29.67)\end{array}$ & 65.09 \\
\hline & & 1500 & $\begin{array}{c}5.27 \\
(27.33)\end{array}$ & 67.85 \\
\hline & & 2000 & $\begin{array}{c}5.05 \\
(25.00)\end{array}$ & 70.59 \\
\hline $\mathbf{T}_{7}$ & Control & & $\begin{array}{c}9.25 \\
(85.00)\end{array}$ & \\
\hline \multicolumn{3}{|c|}{ S. Em. \pm} & 0.14 & \\
\hline \multicolumn{3}{|c|}{ C. D. at $5 \%$} & 0.41 & \\
\hline \multicolumn{3}{|c|}{ C. V. (\%) } & 4.03 & \\
\hline
\end{tabular}

@ Mean of three replications

* Figures outside parenthesis are $\sqrt{\mathrm{x}}+0.5$ transformed value

** Figures in parenthesis are original values 


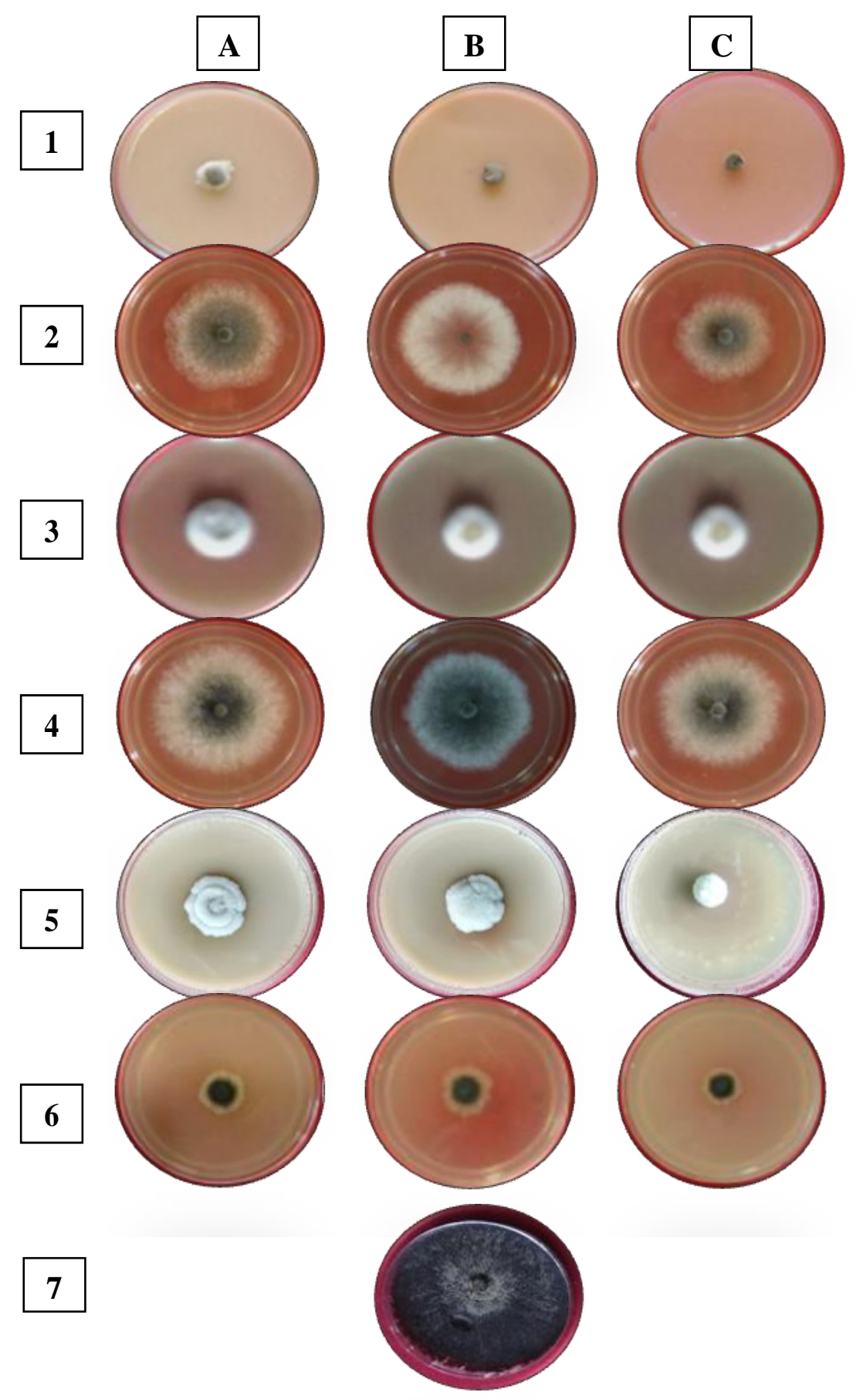

Fungicides

1. Carbendazim $50 \mathrm{WP} \quad$ 5. Hexaconazole $5 \mathrm{EC}$

Concentrations

2. Tebuconazole 25.9 EC 6. Thiophanate methyl $70 \mathrm{WP}$

3. Propiconazole 25 EC 7. Control

A.50ppm

B. $250 \mathrm{ppm}$

4. Azoxystrobin $23 \mathrm{SC}$

C. $500 \mathrm{ppm}$

Plate.1 Growth inhibition of $M$. phaseolina at different concentrations of systemic fungicides 

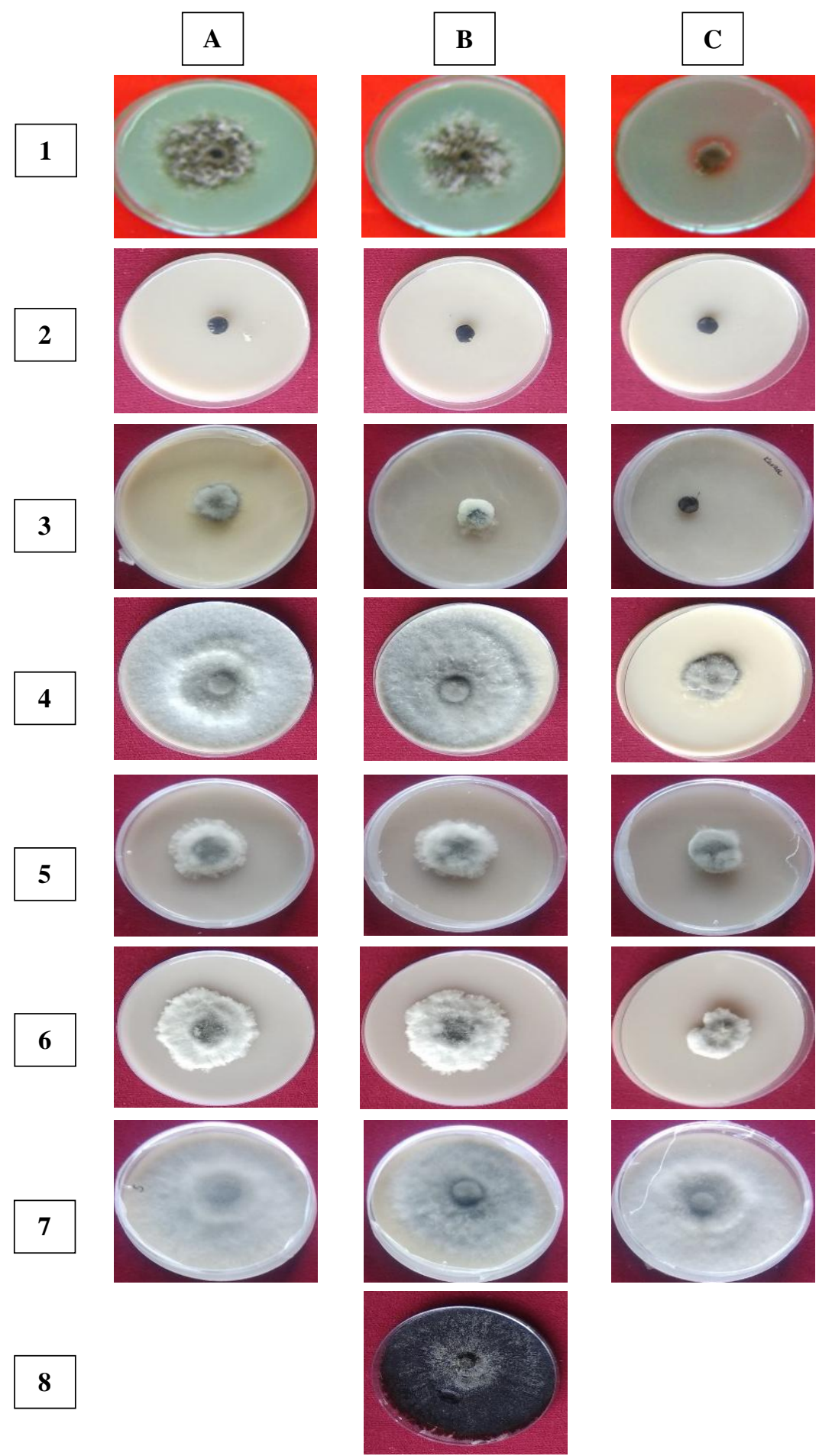

Fungicides

1. Copper oxychloride $50 \mathrm{WP}$

5. Zineb $75 \mathrm{WP}$

Concentrations

2. Mancozeb 75 WP

6. Propineb $70 \mathrm{WP}$

A. $1000 \mathrm{ppm}$

B. $2000 \mathrm{ppm}$

3. Chlorothalonil $75 \mathrm{WP}$

7. Sulphur $80 \mathrm{WDG}$

C. $2500 \mathrm{ppm}$

4. Thiram $75 \mathrm{WP}$

8. Control

Plate.2 Growth inhibition of $M$. phaseolina at different concentrations of non-systemic fungicides 

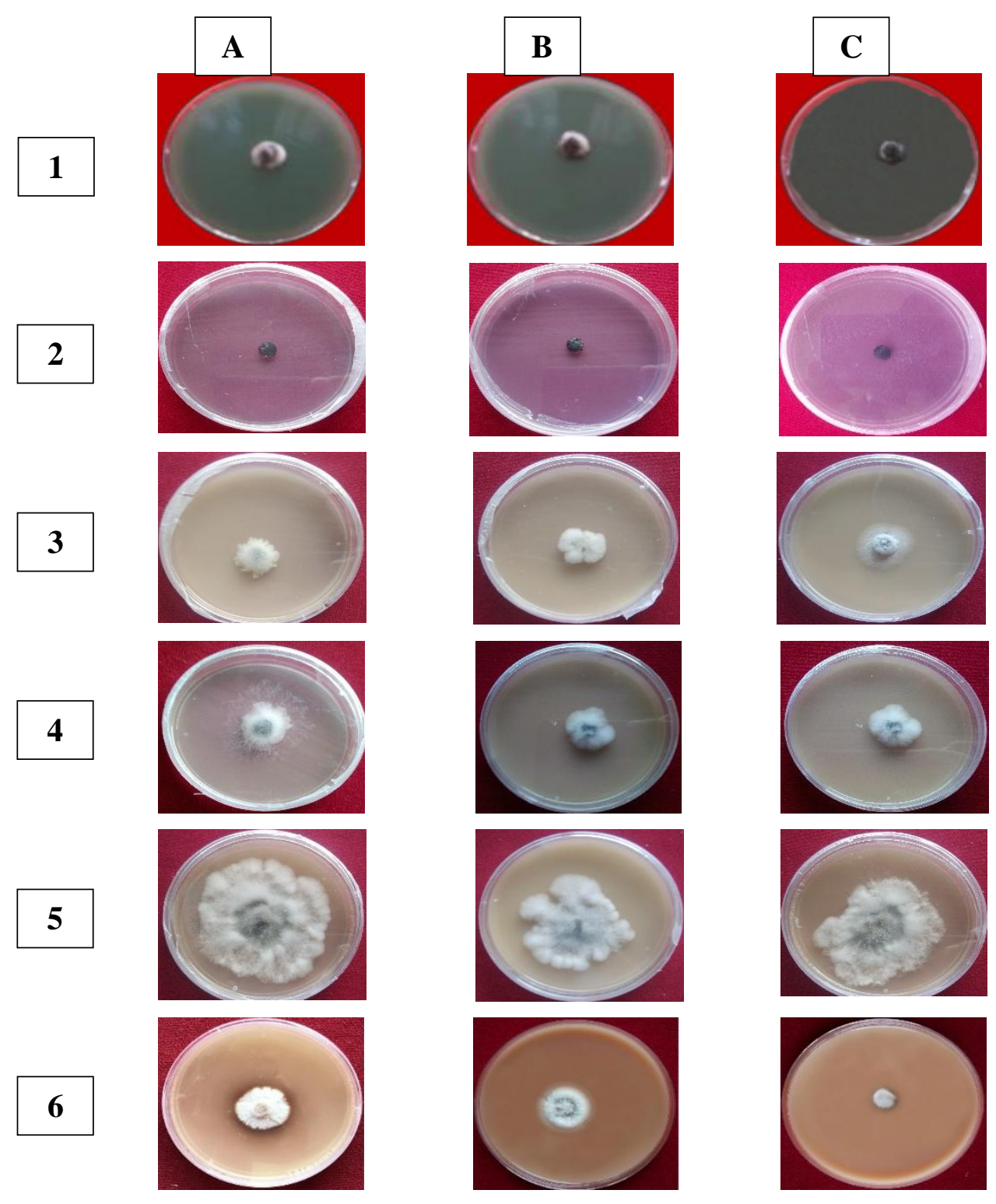

\section{7}

Fungicides

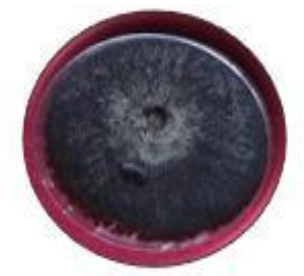

1. Carbendazim 12\% + Mancozeb 63\% WP

Concentrations

2. Carboxin $37.5 \%+$ Thiram $37.5 \% \mathrm{WP}$

A. 500ppm

B. $1500 \mathrm{ppm}$

C. $2000 \mathrm{ppm}$

3. Captan $70 \%+$ Hexaconazole $5 \%$ WP

4. Pyraclostrobin $12.5 \%+$ Epoxiconazole $4.7 \%$ SE

6. Pyraclostrobin 5\% + Mitiram 55\%

5. Hexaconazole $4 \%+$ Zineb $68 \%$

7. Control

Plate.3 Growth inhibition of $M$. phaseolinaat different concentrations of ready-mix fungicides 
At 1500 ppm concentration, highest per cent growth inhibition over control was recorded $(85.88 \%)$ in carbendazim $12 \%+$ mancozeb $63 \%$ followed by carboxin $37.5 \%+$ thiram $37.5 \%(83.14 \%)$. The remaining fungicides viz., pyraclostrobin $5 \%+$ mitiram $55 \%$ $(67.85 \%)$, captan $70 \%+$ hexaconazole $5 \%$ $(70.98 \%)$ and pyraclostrobin $5 \%+$ mitiram $55 \%(67.85 \%)$ were moderately effective against $M$. phaseolina.

At 2000 ppm concentration, highest per cent growth inhibition over control was recorded $(88.24 \%)$ in carbendazim $12 \%+$ mancozeb $63 \%$ followed by carboxin $37.5 \%+$ thiram $37.5 \%$, $(83.14 \%)$. The remaining fungicides viz., pyraclostrobin $5 \%+$ mitiram $55 \%$ $(70.59 \%)$, captan $70 \%+$ hexaconazole $5 \%$ $(76.47 \%)$ and pyraclostrobin $5 \%+$ mitiram $55 \%(59.21 \%)$ were moderately effective against $M$. phaseolina.

Different fungicides greatly varied in their efficacy to inhibit the growth of fungus under study. The growth inhibition per cent positively correlated with increase in concentration for all the chemicals tested. It is inferred from results that there was less mycelial growth of the pathogen in carbendazim $12 \%+$ mancozeb 63\%and carboxin $37.5 \%$ + thiram $37.5 \%$ at 2000 ppm and also significantly lesser growth at 500 and $1500 \mathrm{ppm}$ compared to the Thus, both the fungicides proved the most effective for $M$. phaseolina.

The results are in accords with work of the Maruti et al., (2017) found that carbendazim $12 \%+$ mancozeb $63 \% \mathrm{WP}$, carboxin $37.5 \%+$ thiram $37.5 \%$ WP showedcent per cent of inhibition of $R$. bataticola at all the concentrations i.e., $0.10 \%, 0.20 \%$ and $0.30 \%$. Sangappa and Mallesh (2016) observed that carbendazim $12 \%$ + mancozeb $63 \%$, showed complete inhibition of mycelial growth at all the concentrations, i.e., $0.05,0.10$ and $0.2 \%$.
Results concluded that among the systemic fungicides the minimum mycelial growth was recorded in carbendazim and thiophanate methyl at $500 \mathrm{ppm}$ and also significantly lesser growth at 50 and $250 \mathrm{ppm}$ compared to rest of concentrations. In case of non-systemic fungicides, mancozeb and chlorothalonil proved maximum growth inhibition of $M$. phaseolina at $2500 \mathrm{ppm}$ and also significantly lesser growth at 1000 and 2000 ppm.

Among ready mix fungicides at various concentrations were screened in vitro against M. phaseolina, in Carbendazim (12\%) + Mancozeb (63\%) and Carboxin (37.5\%) + Thiram $(37.5 \%)$ were found highest growth inhibition at $2000 \mathrm{ppm}$ and also significantly lesser growth at 500 and 1500 ppm compare with other fungicides.

\section{References}

Anonymous 2018. Department of Agriculture and Cooperation, Ministry of Agriculture, Government of India.

Chattopadhyay, C. and Kalpana Sastry, R. 2002. Combining viable disease controls tools for management of sesame stem rot caused by Macrophomina phaseolina (Tassi) Goid. Indian Journal of Plant Protection, 30(2): 132-138.

Dhingra, O. D. and Sinclair, J. B. 1995. Basic Plant Pathology Methods. $2^{\text {nd }}$ Ed. Lewis Publishers, USA. pp.434.

Ebenezar, E. G. and Wesely, E. G. 2000. Effect of fungicides on Macrophomina phaseolina causing root rot in greengram. Pestology, 24(3):12-14.

Khan, M. A. and Gangopadhyay, S. 2008. Efficacy of Pseudomonas fluorescens in controlling root rot of chickpea caused by Macrophomina phaseolina. Indian Journal of Mycology and Plant Pathology, 38(3): 580-587.

Kumari, R. and Shekhawat, K. S. 2012. 
Integrated management against root-rot of mungbean (Vigna radiata L. Wilczek) incited by Macrophomina phaseolina. Indian Journal Plant Pathology Microbiology, 3(5): 215-747. Maruti, Savitha, A.S., Sunkad, G and Amaresh, Y. S. 2017. In vitro efficacy of fungicides and bioagents against dry root rot of pigeonpea caused by Rhizoctonia bataticola (Taub.) Butler. International Journal of Pure and Applied Sciences, 5(6): 1341-1347.

Nene, Y. L., Shelia, V. K. and Sharma, S. B. 1996. A World List of Chickpea and Pigeonpea Pathogens ( $5^{\text {th }}$ Edition), International Crop Research Institute for Semi-Arid Tropics, Patancheru, Andhra Pradesh.

Pande, S. and Sharma, M. 2010. Climate Change: Potential Impact on Chickpea and Pigeonpea Diseases in the Rainfed Semi-Arid Tropics. In 5th International Food Legumes Research Conference (IFLRCV) \& 7th European Conference on Grain Legumes (AEP VII) April 2630, Antalya, Turkey.
Ravichandran, S. and Hegde, Y. R. 2017. Management of dry root rot of chickpea caused by Rhizoctonia bataticola through fungicides. Int.J.Curr.Microbiol.App.Sci. 6(7): 59416.

Sangappa, G. and Mallesh, S. B. 2016. Effect of bioagents and chemicals for the management of aerial blight and dry root rot of black gram incited by Rhizoctonia bataticola. International Journal of Plant Protection, 9(2): 424429.

Sharma, M. and Pande, S. 2013. Unraveling effects of temperature and soil moisture stress response on development of dry root rot Rhizoctonia bataticola (Taub.) in chickpea. American Journal of Plant Sciences, 4: 584-589.

Taya, R. S., Tripathi, N. N. and Panwar, M. S. 1988. Influence of soil type, soil moisture and fertilizers on the severity of chickpea dry root rot caused by Rhizoctonia bataticola (Taub.) Butler. Indian Journal of Mycology and Plant Pathology, 18: 133-136.

\section{How to cite this article:}

Lokesh. R., K. B. Rakholiya and Thesiya. M. R. 2020. Evaluation of Different Fungicides against Macrophomina phaseolina (Tassi) Goid. Causing Dry Root Rot of Chickpea (Cicer arietinum L.) invitro. Int.J.Curr.Microbiol.App.Sci. 9(07): 901-911. doi: https://doi.org/10.20546/ijcmas.2020.907.105 